\title{
EXACT NUMERICAL CALCULATION OF CHROMATICITY IN SMALL RINGS
}

\author{
ALEX J. DRAGT \\ Center for Theoretical Physics, Department of Physics and Astronomy, University of Maryland, \\ College Park, Maryland 20742 USA
}

(Received October 6, 1981; in final form August 23, 1982)

\begin{abstract}
A purely numerical method, which is both conceptually simple and exact, has been developed for chromaticity calculations. Its use can therefore serve as a benchmark for checking other methods. The method employs a numerical integration procedure which simultaneously integrates the equations of motion for a particle trajectory and the variational equations for neighboring trajectories. A rapidly convergent Newton's search procedure is used to find closed orbits, and the solution to the variational equations provides the tunes of these orbits. It is found that the natural chromaticity of a small ring can vary widely over the tune diagram; and, contrary to common lore, can even be positive. It is also found that nonlinear dipole contributions can be very important for small rings. Finally, it is found that fringe fields, even in the hard edge approximation, can have nonlinear effects which influence chromaticities. Consequently, methods of chromaticity calculation which treat dipoles and fringe fields in the linear transfer matrix approximation are not expected to be correct for small rings.
\end{abstract}

\section{INTRODUCTION}

Chromaticity correction and control is often essential for the operation of synchrotrons and storage rings. ${ }^{1}$ Methods for the calculation of chromaticity have been developed by several authors. They range from completely analytical calculations to hybrid calculations that make use of analytical results combined with numerical results from matrix lattice codes. ${ }^{2}$

Chromaticity arises from two effects. First, for a given location, the bending and focussing strengths of dipoles and quadrupoles (and all other elements as well) are momentum dependent. Second, the location, size, and shape of the closed orbit for each momentum do, in fact, depend upon the momentum. This second effect is particularly subtle because it influences betatron oscillations about each closed orbit in a variety of ways. A list of these ways, perhaps not exhaustive, is as follows:

a) The total path length of a closed orbit depends upon momentum.

b) If a closed orbit passes through a nonuniform field region, the gradient of the field, which controls betatron oscillations, depends on the location of the orbit. For example, an orbit passing through a sextupole field experiences, in effect, a position dependent quadrupole field. In gen- eral, particles with different momenta may experience different focussing because they pass through different magnetic fields.

c) There are various terms in the equations of motion which are effectively nonlinear if the orbit has large curvature. Such terms are particularly important in dipoles with small bending radii. Some of these terms have been discussed in the case of a combined function lattice. ${ }^{3}$ The effect of these nonlinear terms is similar to that of nonuniform fields.

d) Fringe field focussing depends on the angles of entry and exit, and these angles in turn depend on momentum because the shape of the design orbit depends on momentum.

It is not clear that all these effects have been incorporated into existing methods of chromaticity calculation. Moreover, considering the subtlety of the effects already listed, it is not clear that all possible effects have been completely identified. Indeed, differing existing methods have been applied in some cases to the same problem with differing results. It is shown in Appendix A, for example, that nonlinear dipole contributions are important for small rings. Similarly, Appendix B illustrates the importance of treating fringe field effects properly.

For these reasons, a new method of chromaticity calculation that is both conceptually simple 
and exact has been developed. Its use can therefore serve as a benchmark for checking other methods. There is one caveat, however. The method to be presented requires the direct numerical integration of orbits. This is no problem for relatively small machines with simple lattices, but could conceivably be expensive in some cases for large machines with complex lattices.

The methods of this paper grew out of a study of various proposed lattice designs for a proton storage ring to be built at the Los Alamos National Laboratory. Although chromaticity correction will probably not prove to be a problem for this machine, all calculations will be carried out for this machine in order to illustrate the method. Other simple lattices, such as those relevant to synchrotron light sources, can be treated with equal ease.

Section 2 of this paper describes the general method of computation. Specific application is made to two particular lattice designs in Section 3. Section 4 provides a concluding summary.

\section{METHOD OF COMPUTATION}

Briefly stated, the method of chromaticity calculation to be employed is as follows:

1. Specify the machine lattice including dipole strengths, quadrupole strengths, sextupole strengths, etc.

2. Specify the momentum of a test particle.

3. Find the closed orbit corresponding to this momentum.

4. Find the tunes of this closed orbit.

5. Repeat steps 2 through 4 for a range of momentum values, observe how tunes vary with particle momentum, and thereby determine the chromaticity.

Obviously, the key elements in this procedure are steps 3 and 4 . They are carried out with the aid of a numerical integration code which simultaneously integrates the equation of motion for a particle trajectory and the variational equations for neighboring trajectories. ${ }^{4,5}$ All together, a total of 20 first order equations ( 4 for the main orbit and 16 variational) are integrated.

As indicated elsewhere, closed orbits can be found very efficiently by use of the variational equations coupled with Newton's method contraction maps and the concept of Poincare surfaces of section. ${ }^{6}$ Once a closed orbit has been found, the tunes for this closed orbit are also given by the solution of the variational equations. ${ }^{7}$

As a check on the correctness and accuracy of the numerical integration procedure, the momentum is first taken to have the design value. Then the orbit and variational equations are integrated for the design orbit associated with the lattice. For the specified design momentum, the design orbit is the orbit that consists of circular arcs in dipoles, and straight lines (on axis) in all other lattice elements. Integration of the orbit equations in this case should yield just this simple orbit, and this fact serves as a check on the numerical integration method. In addition, simultaneous integration of the variational equations, in turn, provides the two tune values associated with the design orbit. These tune values should agree with those provided by matrix methods, and this comparison serves as a second check on the integration method.

Next, a momentum value is selected somewhat off from the design value. Let $p^{0}$ denote the design momentum, and $p$ the momentum value of interest. Write

$$
p=p^{0}(1+\delta)
$$

and give $\delta$ a small value. Once the closed orbit for this $\delta$ value has been computed, comparison of the closed off-momentum orbit with the design orbit provides the $\eta$ and $\eta^{\prime}$ functions for the lattice to all orders in $\delta$. In particular the $\eta$ and $\eta^{\prime}$ functions found in this way, in the limit of vanishing $\delta$, should agree with those provided by matrix methods. This comparison serves as a third check on the integration method.

Finally, simultaneous integration of the variational equations for the off-momentum closed orbit provides the tune values for this orbit. Comparison of these tune values with those of the design orbit gives chromaticities to all orders in $\delta$.

\section{APPLICATIONS}

In this section the general methods of the previous section will be applied to two specific lattices which are under consideration for a Proton Storage Ring (PSR) to be built at the Los Alamos National Laboratory. Detailed numbers, with an excessive number of significant figures, will be presented in order to provide benchmark results 


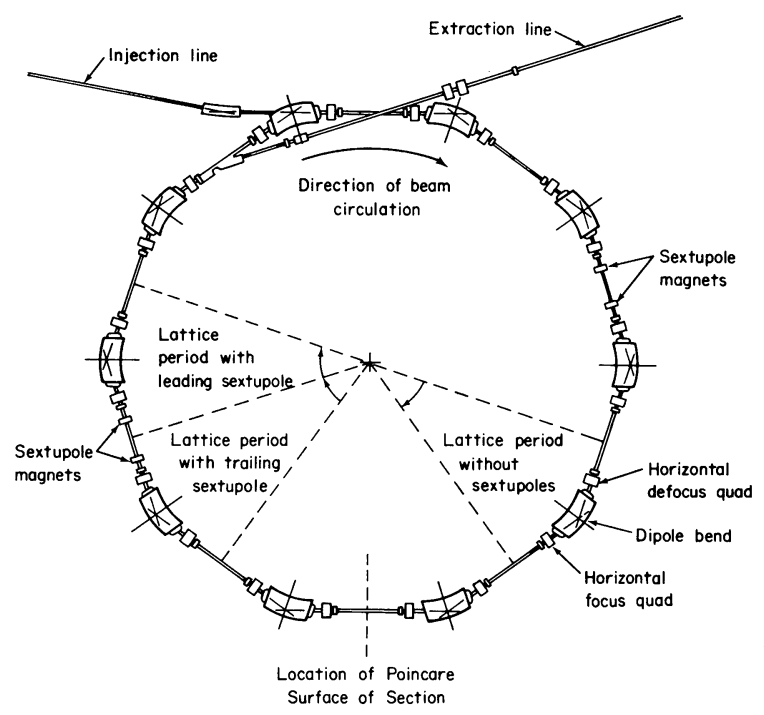

FIGURE 1 Proposed lattice design for Proton Storage Ring. The ring is shown as it would appear looking down from above.

for comparison with other methods of computation. ${ }^{4}$

The magnets in the PSR will be arrayed in a ten-sided separated function lattice consisting of straight sections and bends. See Fig. 1. The lattice is composed entirely of dipoles, quadrupoles, and drifts with the exception of two pairs of sextupoles. When these sextupoles are turned off, the lattice has ten identical periods. When the sextupoles are turned on, the symmetry of the lattice is reduced. Inspection of Fig. 1 shows that in this latter case the lattice can be viewed as consisting of two identical halves, and thus it has two identical periods.

Two possible choices are currently being considered for the type of dipole bend magnet to be used. One possibility is to use bend magnets whose faces are arranged to be normal to the onmomentum design orbit as illustrated in Fig. 2. A second possibility, illustrated in Fig. 3, is to use bend magnets whose faces are parallel. Orbit calculations will be presented for both kinds of bend magnets.

The PSR, which is to be filled by the LAMPF linear accelerator, is designed to have a circulation time $\tau$ which is the 72 'nd multiple of the LAMPF linac rf period,

$$
\begin{aligned}
\tau & =(72) /\left(201.25 \times 10^{6}\right) \\
& =357.764 \text { nanoseconds }
\end{aligned}
$$

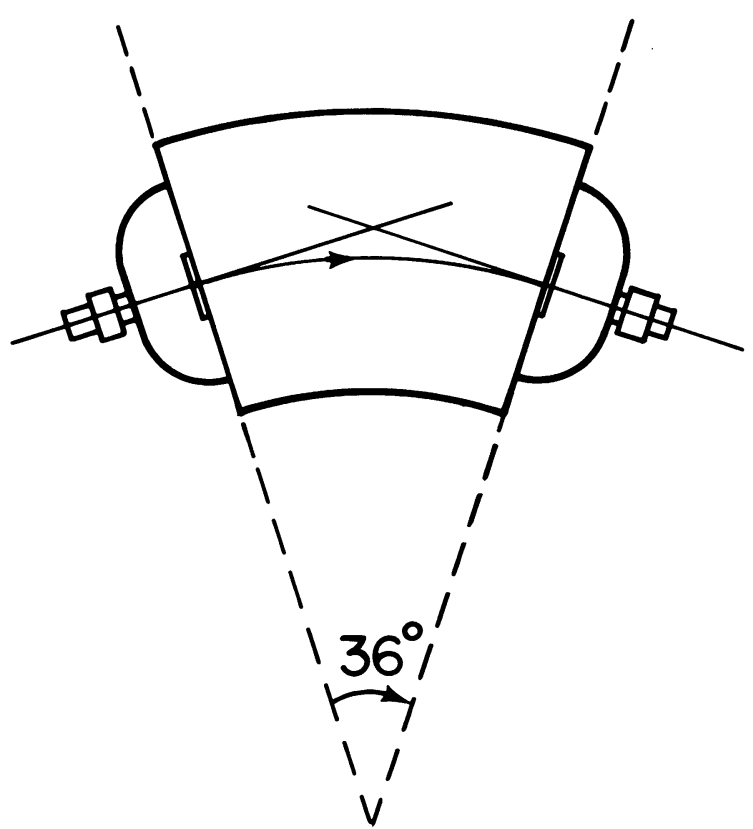

FIGURE 2 Ring configuration for which the faces of each dipole bending magnet are normal to the design orbit. Trajectories are integrated using cartesian coordinate systems in all sections except bends, and cylindrical coordinate systems are used in bends.

The second key parameter which specifies the dimensions of the PSR is the kinetic energy of the on-momentum design closed orbit. This energy is assumed to have the Boeing value

$$
E=797.000 \mathrm{MeV} \text {. }
$$

Protons of this energy have a velocity $v$ given by

$$
v=2.521886 \times 10^{8} \text { meters } / \text { second }
$$

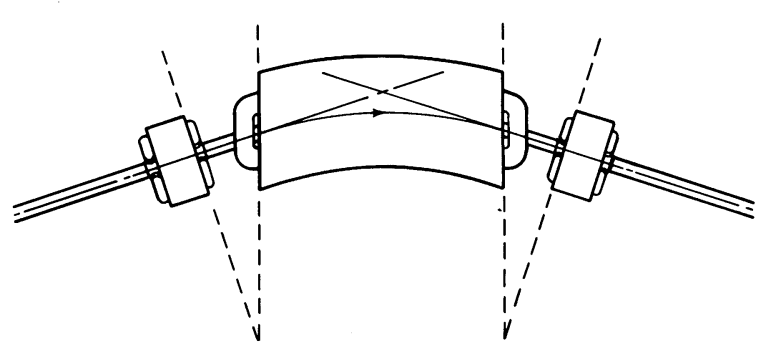

FIGURE 3 Ring configuration for which the faces of each dipole bending magnet are parallel. Trajectories are integrated using cartesian coordinates in the long straight sections, in quadrupoles and sextupoles, and in the bend magnets; and small, wedge shaped patches of cylindrical coordinates are used in the short straight sections between bends and quadrupoles. 
Consequently, the PSR closed on-momentum design orbit has a circumference $C$ given by the relation

$$
C=v \tau=90.2240 \text { meters. }
$$

The lengths and kinds of the various lattice elements which combine to make up this circumference are listed in Table I and shown in Fig. 4. Three typical lattice periods are displayed, and from them the entire lattice can be assembled. See Fig. 1. Note that the length specified for the dipole is not its physical length, but rather the length of the on-momentum design orbit within it. The radius of curvature $\rho_{0}$ of the design orbit within the dipole is given by

$$
\begin{aligned}
\rho_{0}=(5 / \pi)(2.54948) \\
=4.057623443139 \cdots \text { meters } .
\end{aligned}
$$

The strength $B_{z}{ }^{0}$ of the dipole field is taken to be given exactly by the equation

$$
B_{z}{ }^{0}=1.2 \text { Tesla }
$$

Hence, the rigidity of the design on-momentum orbit is given by the relation

$$
\begin{aligned}
B_{z}^{0} \rho_{0} & =(1.2)(5 / \pi)(2.54948) \\
& =4.86914813176 \cdots \text { Tesla meters } .
\end{aligned}
$$

One last concern needs to be mentioned. Due to fringe fields, dipole magnets unavoidably provide vertical focussing or defocussing. In the case of normal entry magnets, there is no vertical focussing effect on the design orbit since it does indeed enter and exit bend magnets normally. However, there is an effect on off-momentum orbits since they do not enter and exit dipoles normally; and this effect must be taken into account in calculating chromaticities. In the case of parallel faced bend magnets, all trajectories of interest experience vertical fringe field focussing. Appendix B gives a numerical example of the importance of treating fringe field focussing in a manner that goes beyond the usual matrix formulation.

It should also be pointed out that since the methods of this paper employ exact numerical integration of trajectories, no correction due to rotated pole edges is required for the horizontal

\begin{tabular}{|c|c|c|}
\hline \multicolumn{3}{|c|}{ Lattice period without sextupoles } \\
\hline Element & Length & Strength \\
\hline drift & $2.28646 \mathrm{~m}$ & - \\
\hline $\begin{array}{l}\text { hor defocus } \\
\text { quad }\end{array}$ & $0.5 \mathrm{~m}$ & variable \\
\hline drift & $0.45 \mathrm{~m}$ & - \\
\hline edge & & $0^{\circ}$, or $18^{\circ}=\pi / 10 \mathrm{rad}$ \\
\hline bend & $2.54948 \mathrm{~m}$ & 1.2 Tesla \\
\hline edge & 0 & $0^{\circ}$, or $18^{\circ}=\pi / 10 \mathrm{rad}$ \\
\hline drift & $0.45 \mathrm{~m}$ & - \\
\hline $\begin{array}{l}\text { hor focus } \\
\text { quad }\end{array}$ & $0.5 \mathrm{~m}$ & variable \\
\hline $\begin{array}{l}\text { drift } \\
\text { Total }\end{array}$ & $\frac{2.28646 \mathrm{~m}}{9.02240 \mathrm{~m}}$ & - \\
\hline
\end{tabular}
motion. This correction which is purely geometric, must be included, or course, when matrix

\begin{tabular}{|c|c|c|}
\hline $\begin{array}{l}\text { Element } \\
\text { drift }\end{array}$ & $\begin{array}{l}\text { Length } \\
2.28646 \mathrm{~m}\end{array}$ & Strength \\
\hline $\begin{array}{l}\text { hor defocus } \\
\text { quad }\end{array}$ & $0.5 \mathrm{~m}$ & variable \\
\hline drift & $0.45 \mathrm{~m}$ & - \\
\hline edge & 0 & $0^{\circ}$, or $18^{\circ}=\pi / 10 \mathrm{rad}$ \\
\hline bend & $2.54948 \mathrm{~m}$ & 1.2 Tesla \\
\hline edge & 0 & $0^{\circ}$, or $18^{\circ}=\pi / 10 \mathrm{rad}$ \\
\hline drift & $0.45 \mathrm{~m}$ & - \\
\hline $\begin{array}{l}\text { hor focus } \\
\text { quad }\end{array}$ & $0.5 \mathrm{~m}$ & variable \\
\hline drift & $0.3 \mathrm{~m}$ & - \\
\hline $\begin{array}{l}\text { horiz chrom } \\
\text { sext }\end{array}$ & $0.5 \mathrm{~m}$ & variable \\
\hline $\begin{array}{l}\text { drift } \\
\text { Total }\end{array}$ & $\frac{1.48646 \mathrm{~m}}{9.02240 \mathrm{~m}}$ & - \\
\hline
\end{tabular}
methods are used.
TABLE I

\begin{tabular}{|c|c|c|}
\hline Element & Length & Strength \\
\hline drift & $1.48646 \mathrm{~m}$ & - \\
\hline $\begin{array}{l}\text { vert chrom } \\
\text { sext }\end{array}$ & $0.5 \mathrm{~m}$ & variable \\
\hline drift & $0.3 \mathrm{~m}$ & - \\
\hline $\begin{array}{l}\text { horiz defocus } \\
\text { quad }\end{array}$ & $0.5 \mathrm{~m}$ & variable \\
\hline drift & $0.45 \mathrm{~m}$ & 一 \\
\hline edge & 0 & $0^{\circ}$, or $18^{\circ}=\pi / 10 \mathrm{rad}$ \\
\hline bend & $2.54948 \mathrm{~m}$ & 1.2 Tesla \\
\hline edge & 0 & $0^{\circ}$, or $18^{\circ}=\pi / 10 \mathrm{rad}$ \\
\hline drift & $0.45 \mathrm{~m}$ & - \\
\hline $\begin{array}{l}\text { horiz focus } \\
\text { quad }\end{array}$ & $0.5 \mathrm{~m}$ & variable \\
\hline $\begin{array}{l}\text { drift } \\
\text { Total }\end{array}$ & $\frac{2.28646 \mathrm{~m}}{9.02240 \mathrm{~m}}$ & - \\
\hline
\end{tabular}

PSR Lattice Parameters

Lattice period with trailing sextupole

Lattice period with leading sextupole

The reader should now have sufficient background to evaluate numerical results. Table II presents the results of five computer runs for the case of the PSR with normal entry bend magnets. The first run is for the design orbit, and the re- 


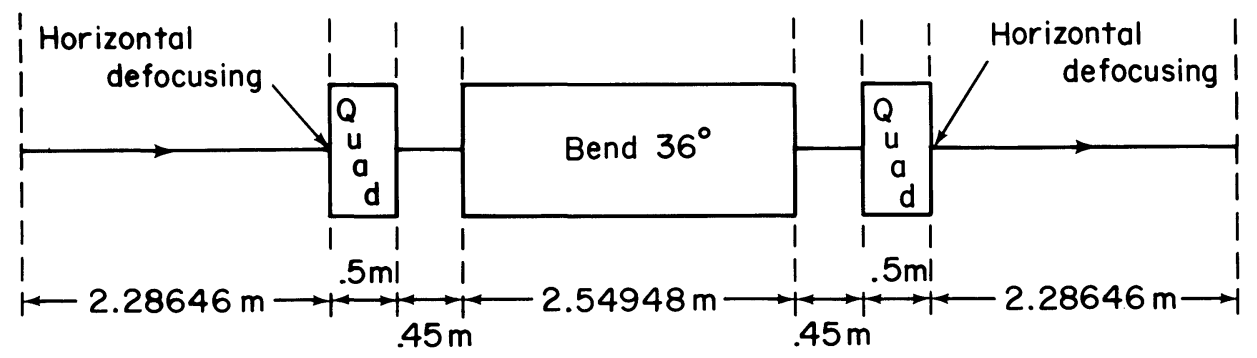

Period without sextupoles

Period with

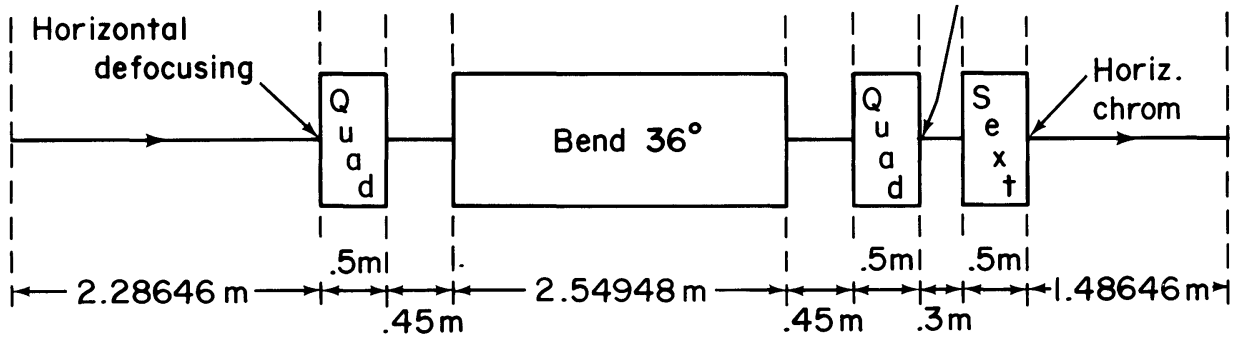
trailing sextupole

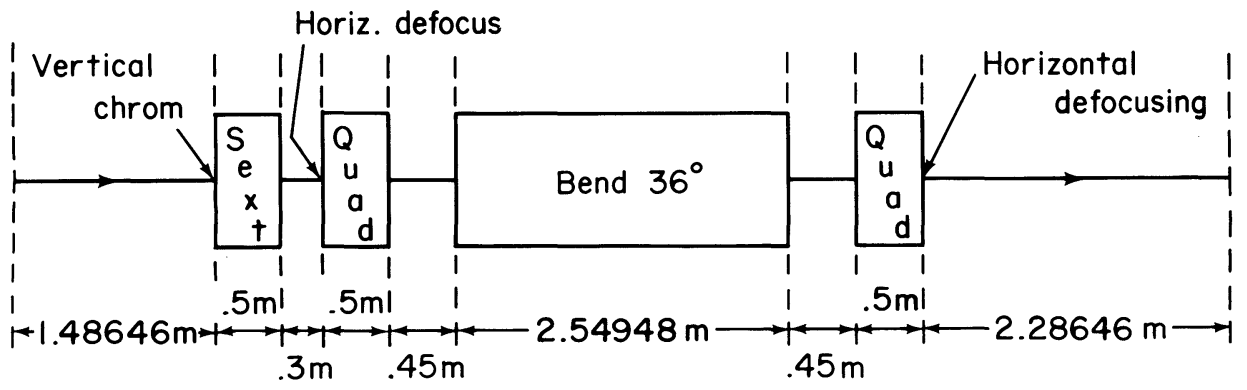

Period with leading sextupole

FIGURE 4 Details and Dimensions of Proton Storage Ring lattice showing three typical periods.

maining four are for off-momentum orbits. The range $\delta= \pm 10^{-3}$ is expected to be typical for the PSR.

A word is in order about units. The computer program itself employs dimensionless units. Momenta are measured in units of $p^{0}$, and distances are measured in units of $C$. The variables $Q_{y}, P_{y}$ refer to the closed orbit initial conditions for the horizontal degree of freedom. Here the Poincare surface of section is taken to be the beginning of the lattice period just preceding the lattice period with a trailing sextupole. See Figs. 1 and 4 . Because of assumed midplane symmetry, both the initial coordinate and momentum for the vertical degree of freedom are always zero, and therefore need not be shown.

TABLE II

Selected Closed Orbit Data for PSR with Normal Entry Bend Magnets

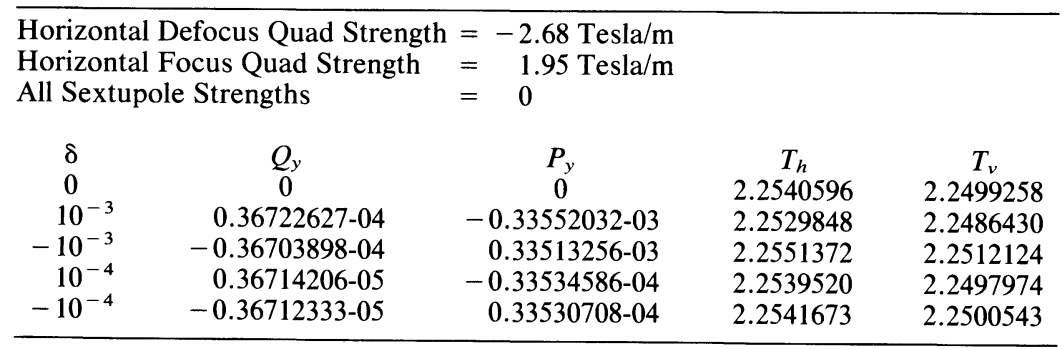


Inspection of the table shows that the initial conditions for the closed orbit depend on $\delta$ as expected. In general, one has an expansion of the form

$$
\begin{aligned}
& Q_{y}(\delta)=\delta Q_{y}{ }^{\prime}(0)+\left(\delta^{2} / 2\right) Q_{y}{ }^{\prime \prime}(0)+\cdots \\
& P_{y}(\delta)=\delta P_{y}{ }^{\prime}(0)+\left(\delta^{2} / 2\right) P_{y}{ }^{\prime \prime}(0)+\cdots
\end{aligned}
$$

It follows that

$$
\begin{aligned}
& Q_{y}{ }^{\prime}(0)=\left[Q_{y}(\delta)-Q_{y}(-\delta)\right] /(2 \delta)+0\left(\delta^{2}\right) \\
& P_{y}{ }^{\prime}(0)=\left[P_{y}(\delta)-P_{y}(-\delta)\right] /(2 \delta)+0\left(\delta^{2}\right) \\
& Q_{y}{ }^{\prime \prime}(0)=\left[Q_{y}(\delta)+Q_{y}(-\delta)\right] / \delta^{2}+0\left(\delta^{2}\right) \\
& P_{y}{ }^{\prime \prime}(0)=\left[P_{y}(\delta)+P_{y}(-\delta)\right] / \delta^{2}+0\left(\delta^{2}\right)
\end{aligned}
$$

Using the data of Table II for the cases $\delta=$ $\pm 10^{-4}$, one finds from Eqs. (3.9) and (3.10) the results

$$
\begin{aligned}
& Q_{y}{ }^{\prime}(0)=3.6713269 \times 10^{-2} \\
& P_{y}{ }^{\prime}(0)=-3.3532647 \times 10^{-1} \\
& Q_{y}{ }^{\prime \prime}(0)=1.873 \times 10^{-2} \\
& P_{y}{ }^{\prime \prime}(0)=-3.878 \times 10^{-1}
\end{aligned}
$$

At this point, it is possible to make a comparison with the results of a linear matrix code. First, the tunes of the design orbit, as computed using a linear matrix code, should agree with the tunes listed in Table II for the case $\delta=0$. This comparison has been made, and agreement is found to at least all digits presented. Second, when account is taken of the dimensionless units employed, one expects to have for an off-momentum orbit the relations

$$
\begin{aligned}
\eta & =Q_{y}{ }^{\prime}(0) C=3.3124180 \text { meters } \\
\eta^{\prime} & =P_{y}{ }^{\prime}(0)=-3.3532647 \times 10^{-1}
\end{aligned}
$$

Here the $\eta$ and $\eta^{\prime}$ functions are to be calculated at the entrance to a lattice period. [Note that due to customary use and abuse of notation, the primes on the left and right sides of Eq. (3.13b) have different meanings.] It has been verified that the $\eta$ and $\eta^{\prime}$ functions as given by a linear matrix code do indeed satisfy Eq. (3.13) to at least 8 significant figures. Thus, the numerical method of this paper passes all the tests described in section 2.

When the values Eqs. (3.11) and (3.12) are inserted into Eq. (3.8) for the case $\delta=10^{-3}$, one finds that the quadratic terms in Eq. (3.8) make a small contribution of much less than a percent. Comparing the values thus obtained for $Q_{y}(\delta)$ and $P_{y}(\delta)$ with those shown in Table II, one obtains agreement to 7 significant figures. Thus, terms in the off-momentum orbit higher than quadratic in $\delta$ are completely negligible for the PSR.

Examination of the tune values listed in Table II shows that the tunes for the design orbit and the off-momentum closed orbits are not the same. Thus, the chromaticities are not zero. In general one has an expansion for tunes of the form

$$
\begin{aligned}
& T_{h}(\delta)=T_{h}(0)+\delta T_{h^{\prime}}(0)+\left(\delta^{2} / 2\right) T_{h^{\prime \prime}}(0)+\cdots \\
& T_{v}(\delta)=T_{v}(0)+\delta T_{v}^{\prime}(0)+\left(\delta^{2} / 2\right) T_{v}{ }^{\prime \prime}(0)+\cdots
\end{aligned}
$$

Here the subscripts $h$ and $v$ refer to horizontal and vertical. It follows that

$$
\begin{aligned}
& T_{h^{\prime}}{ }^{\prime}(0)=\left[T_{h}(\delta)-T_{h}(-\delta)\right] /(2 \delta)+0\left(\delta^{2}\right) \\
& T_{h}{ }^{\prime}(0)=\left[T_{h}(\delta)+T_{h}(-\delta)-2 T_{h}(0)\right] / \delta^{2}+0\left(\delta^{2}\right),
\end{aligned}
$$

with similar formulas for $T_{v}{ }^{\prime}(0)$ and $T_{v}{ }^{\prime \prime}(0)$. Using the data from Table II for $\delta= \pm 10^{-3}$, one finds from (3.15) and their vertical counterparts the results

$$
\begin{aligned}
& T_{h}{ }^{\prime}(0)=-1.0762 \\
& T_{v}{ }^{\prime}(0)=-1.2847 \\
& T_{h}{ }^{\prime \prime}(0)=2.8 \\
& T_{v}{ }^{\prime \prime}(0)=3.8
\end{aligned}
$$

The quantities $T_{h}{ }^{\prime}(0)$ and $T_{v}{ }^{\prime}(0)$ may be taken as the definition of the first-order natural chromaticities of the lattice. Note that they are both negative when the tunes are near the values 2.25 and the lattice has normal entry bend magnets.

Since the PSR beam energy is well below the lattice transition energy, negative chromaticities are required to damp the head-tail instability. According to Eq. (3.16), the natural chromaticities of the PSR near tunes of 2.25 are negative, 
and no chromaticity correction sextupoles are required. Other computer runs show that the same is true at tune combinations of $T_{h}=3.25$, $T_{v}=2.25 ; T_{h}=3.25, T_{v}=2.75 ;$ and $T_{h}=2.25$, $T_{v}=2.75$.

It is common lore that the natural chromaticities of a lattice are always negative. However, this need not always be the case, at least for small rings. Table III shows the result of computer runs for tune values near $T_{h}=0.5$ and $T_{v}=3.3$. Note that both tunes increase when $\delta=10^{-3}$. Accordingly, both chromaticities are positive in this case. Of course, these tunes are not of interest for an actual machine. However, this example shows that natural chromaticities can vary markedly over a tune diagram, and can even change sign. For further comment, see Appendix A.

Because the PSR is expected to operate below its transition energy and at tune combinations for which both natural chromaticities are negative, no chromaticity correction sextupoles are required in the normal mode of operation. However, it is still of interest to see what sextupole strengths would be required to achieve zero chromaticities. Table IV shows the result of computer runs identical to those of Table II except that appropriate sextupole strengths have been selected to make both chromaticities just slightly positive.

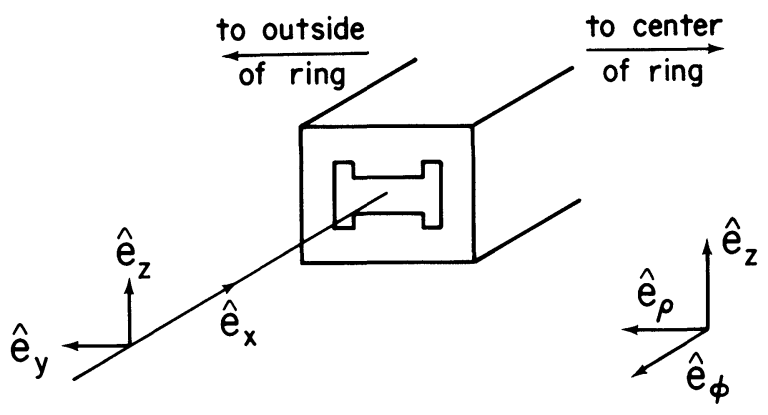

FIGURE 5 Typical choice of cartesian coordinate system for a lattice element. Also shown, to the right, is an associated cylindrical coordinate system unit vector triad.

An explanatory comment is necessary to understand Table IV. The magnetic field in a sextupole is assumed to have the form

$$
\begin{aligned}
& B_{y}=a_{s}(2 y z) \\
& B_{z}=a_{s}\left(y^{2}-z^{2}\right) .
\end{aligned}
$$

Here $y$ lies in the plane of the ring and $z$ is measured perpendicular to the plane of the ring. See Fig. 5. The strength of a sextrupole as listed in the table is given in terms of the coefficient $a_{s}$ which evidently has units of Tesla/(meter) ${ }^{2}$.

With this explanation, it is evident that only

TABLE III

Additional Closed Orbit Data for PSR with Normal Entry Bend Magnets

\begin{tabular}{lcccc}
\hline Horizontal Defocus Quad Strength & $=$ & $-3.5 \mathrm{Tesla} / \mathrm{m}$ & & \\
Horizontal Focus Quad Strength & $=$ & $1.0 \mathrm{Tesla} / \mathrm{m}$ & & \\
All Sextupole Strengths & $=$ & & & \\
\multicolumn{1}{l}{} \\
$\delta$ & $Q_{y}$ & $P_{y}$ & $T_{h}$ & $T_{v}$ \\
0 & 0 & 0 & 0.47460292 & 3.2768393 \\
$10^{-3}$ & $0.70832836-03$ & $-0.58383744-02$ & 0.51971131 & 3.3130274 \\
$-10^{-3}$ & $-0.87445913-03$ & $0.71216148-02$ & 0.41144415 & 3.2323392 \\
\hline
\end{tabular}

TABLE IV

Selected Closed Orbit Data for PSR with Normal Entry Bend Magnets and Activated Sextupoles

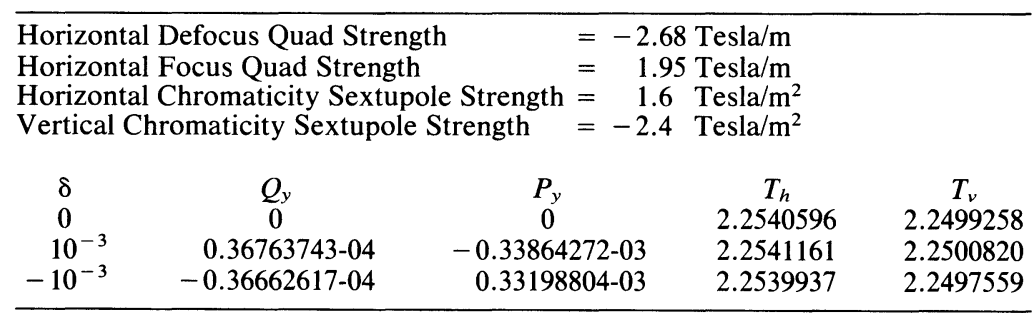


TABLE V

Selected Closed Orbit Data for PSR with Parallel Faced Bend Magnets

\begin{tabular}{|c|c|c|c|c|}
\hline \multicolumn{2}{|c|}{$\begin{array}{l}\text { Horizontal Defocus Quad Strength } \\
\text { Horizontal Focus Quad Strength } \\
\text { All Sextupole Strengths }\end{array}$} & $\begin{array}{l}=-1.92 \mathrm{Tesla} / \mathrm{m} \\
=2.72 \mathrm{Tesla} / \mathrm{m} \\
=0\end{array}$ & & \\
\hline$\delta$ & $Q_{y}$ & $P_{y}$ & $T_{h}$ & $T_{v}$ \\
\hline 0 & 0 & 0 & 2.2541028 & 2.2554377 \\
\hline $10^{-3}$ & $0.36214396-04$ & $-0.34428702-03$ & 2.2531757 & 2.2533289 \\
\hline$-10^{-3}$ & $-0.36198418-04$ & $0.34391480-03$ & 2.2550320 & 2.2575516 \\
\hline $10^{-4}$ & $0.36207211-05$ & $-0.34411955-04$ & 2.2540100 & 2.2552266 \\
\hline$-10^{-4}$ & $-0.36205613-05$ & 0.34408233-04 & 2.2541956 & 2.2556489 \\
\hline
\end{tabular}

TABLE VI

Selected Closed Orbit Data for PSR with Parallel Faced Bend Magnets and Activated Sextupoles

\begin{tabular}{|c|c|c|c|c|c|}
\hline \multicolumn{3}{|c|}{ Horizontal Defocus Quad Strength } & \multicolumn{2}{|c|}{$=-1.92 \mathrm{Tesla} / \mathrm{m}$} & \\
\hline \multicolumn{3}{|c|}{ Horizontal Focus Quad Strength } & & $\mathrm{Tesla} / \mathrm{m}$ & \\
\hline \multicolumn{3}{|c|}{ Horizontal Chromaticity Sextupole Strength = } & $=$ & $\mathrm{Tesla} / \mathrm{m}^{2}$ & \\
\hline \multicolumn{3}{|c|}{ Vertical Chromaticity Sextupole Strength $=$} & $=-3$. & Tesla $/ \mathrm{m}^{2}$ & \\
\hline$\delta$ & $Q_{y}$ & $P_{y}$ & & $T_{h}$ & $T_{v}$ \\
\hline 0 & 0 & 0 & & 2.2541028 & 2.2554377 \\
\hline $10^{-3}$ & $0.36222518-04$ & -0.346840 & $43-03$ & 2.2541486 & 2.2555294 \\
\hline$-10^{-3}$ & $-0.36190533-04$ & 0.341352 & $205-03$ & 2.2540540 & 2.2553242 \\
\hline
\end{tabular}

modest sextupole strengths are required to modify the chromaticity. By comparing Tables II and IV, one sees that the use of sextupoles makes a small change in the location of the off-momentum closed orbit.

The discussion in this section so far has been devoted to the case of bend magnets with normal entry. A similar discussion can be given for the case of bend magnets with parallel faces. Table $\mathrm{V}$ shows the results of runs for a case in which the quadrupole strengths have again been set to achieve tunes near 2.25. Although not shown here, the results of these and other runs have again been compared with matrix codes, and all the tests described in section 2 are passed with at least 8 significant figure accuracy. Note that both natural chromaticities are again negative, e.g., the tunes decrease when $\delta$ is given the value $\delta=10^{-3}$. Using the numbers listed in the table, it is found that the chromaticity coefficients now have the values

$$
\begin{aligned}
& T_{h^{\prime}}(0)=-.92815 \\
& T_{v}{ }^{\prime}(0)=-2.11135 \\
& T_{h^{\prime \prime}}(0)=2.1 \\
& T_{v}^{\prime \prime}(0)=5.1
\end{aligned}
$$

Both chromaticities are also found to be negative at the tune combinations of $T_{h}=3.25, T_{v}=2.25$; $T_{h}=3.25, T_{v}=2.75$; and $T_{h}=2.25, T_{v}=2.75$.

Other numerical work, not shown, indicates that both natural chromaticities are again positive near tune values of $T_{h}=0.5$ and $T_{v}=3.2$.

Finally, the results of computer runs displayed in Table VI show that for a case of actual interest, again only modest although somewhat larger sextupole strengths are required to modify the chromaticity.

\section{CONCLUDING SUMMARY}

Section 2 of this paper indicated that the simultaneous integration of the equations of motion for a particle trajectory, and the variational equations for neighboring trajectories, readily leads to a determination of closed orbits, their tunes, and the variation of tunes with total momentum. These methods were then applied to a particular lattice in section 3. Specific numerical results were provided which can be used to benchmark other methods of chromaticity determination. It was also found that the natural chromaticity of a small ring can vary widely over the tune diagram.

In the course of these calculations, the equa- 
tions of motion in each lattice element were treated exactly. The reader who wishes to gauge the importance of treating nonlinear dipole terms properly in the case of a small ring is referred to Appendix A. There it is shown, at least in the case of the PSR, that a chromaticity calculation which treats dipoles only in the linear transfer matrix approximation gives results which are very far from the correct values. Similarly, Appendix B gives a numerical example of the importance of fringe field effects.

\section{ACKNOWLEDGMENTS}

The author thanks Dr. A. Garren, Dr. R. Servranckx, and Dr. R. K. Cooper for many helpful conversations. He is also indebted to the Accelerator Technology Division of the Los Alamos National Laboratory where this work was begun. He thanks Dr. R. K. Cooper, Dr. G. Lawrence, and Dr. E. Knapp for their fine hospitality. This work was supported in part by the U.S. Department of Energy under contract \#DE-AS0580ER10666.A000.

\section{APPENDIX A}

\section{The Difference between Small and Large Rings in the Calculation of Chromaticities and the Importance of Nonlinear Dipole Contributions}

As mentioned in section 1, nonlinear dipole contributions can be important in determining the chromaticity for small rings. The purpose of this appendix is to comment on two differences between small and large rings in the calculation of chromaticities, and to give a numerical example.

In suitably dimensionless cylindrical coordinates and using the polar angle as an independent variable, the Hamiltonian for motion in a dipole is given by the expression ${ }^{4}$

$$
\begin{aligned}
H^{\text {dipole }}= & -\left[C /\left(2 \pi \rho_{0}\right)\right] \\
& \times\left\{Q_{\rho}\left[(1+\delta)^{2}-P_{\rho}{ }^{2}-P_{z}{ }^{2}\right]^{1 / 2}\right. \\
& \left.-\left(C / \rho_{0}\right)(1 / 2) Q_{\rho}{ }^{2}\right\} .
\end{aligned}
$$

Here $\rho_{0}$ is the radius of curvature of the design orbit. Suppose that this Hamiltonian is expanded in a power series about the on-momentum design orbit given by the equation

$$
Q_{\rho}=Q_{\rho}{ }^{0}=\rho_{0} / C .
$$

Then the expansion of the Hamiltonian will in general contain linear, quadratic, cubic, and still higher order terms. The linear and quadratic terms in the expansion reproduce the results of ordinary linear transfer matrix methods for a bend magnet if all the remaining higher order terms in the Hamiltonian are neglected. The neglect of quartic and still higher order terms is in general justified since they are usually quite small. However, the cubic terms may be important for a small ring, and may produce sextupolelike effects.

This may be seen as follows. Calculation of the cubic term in the expansion shows that it is of the form

cubic term

$$
\propto\left(C / \rho_{0}\right)\left(Q_{\rho}-Q_{\rho}^{0}\right)\left(P_{\rho}^{2}+P_{z}^{2}\right) /(1+\delta) .
$$

Observe that the magnitude of the quantities $(\mathrm{C} /$ $\left.\rho_{0}\right)$ and $\left(P_{\rho}^{2}+P_{z}^{2}\right)$ are pretty much independent of the size of a ring. However, the quantity $\left(Q_{\rho}\right.$ $\left.-Q_{\mathrm{\rho}}{ }^{0}\right)$ is proportional to the expected horizontal displacement from the design orbit in a bend, divided by the circumference $C$ of the ring. Now, the expected horizontal displacement in a bend is pretty much the same independent of the size of the ring. Consequently, the quantity $\left(Q_{\rho}-\right.$ $Q_{\rho}{ }^{0}$ ) is greater for a small ring than a large ring. Therefore, the cubic term may be important for small rings even when its neglect is justified in the case of large rings. Because the term in question is in fact cubic, its effect on chromaticities can be similar to that of sextupoles.

To examine the importance of cubic and still higher order terms in the Hamiltonian for motion in a dipole, a series of orbit calculations have been carried out for two different cases. In the first case, all terms in the dipole Hamiltonian were retained though third order. In the second case, only linear and quadratic terms were retained in the dipole Hamiltonian. (As pointed out earlier, this latter case is equivalent to using ordinary linear transfer matrix methods for a bend.) In both cases, the Hamiltonians for the remaining lattice elements were treated exactly.

These calculations showed that the omission of fourth and higher order terms is unimportant for the PSR. For example, use of the dipole Hamiltonian through third order and use of the exact 
Hamiltonian give differences of at most one unit in the last digit for the orbit data of Table II.

However, the cubic terms are important. Table VII shows selected orbit data calculated in the approximation that only linear and quadratic terms are retained in the dipole Hamiltonian. That is, cubic (and of course still higher order) terms have been omitted. It is evident that the entries in Table II and VII differ in the fourth significant figure.

To study the effect of these differences, suppose the quantities given by Eqs. (3.9), (3.10), and (3.15) are computed from the numerical data of Table VII. One finds the following results:

$$
\begin{aligned}
& Q_{y}{ }^{\prime}(0)=3.6713269 \times 10^{-2} \\
& P_{y}{ }^{\prime}(0)=3.3532647 \times 10^{-1} \\
& Q_{y}^{\prime \prime}(0)=3.757 \times 10^{-2} \\
& P_{y}^{\prime \prime}(0)=-3.642 \times 10^{-1} \\
& T_{h}^{\prime}(0)=-2.0497 \\
& T_{v}{ }^{\prime}(0)=-1.8477 \\
& T_{h}^{\prime \prime}(0)=5.4 \\
& T_{v}{ }^{\prime \prime}(0)=5.2
\end{aligned}
$$

Comparison of the results presented in Eqs. (3.11) and (A4) shows that the $\eta$ and $\eta$ ' functions are unaffected by the omission of cubic terms in the Hamiltonian. This is to be expected, because the absence or presence of terms beyond second order should not affect results that are properly derivable from a linear orbit theory. For the same reason, the tunes of the on-momentum design orbit as given in Tables II and VII are in perfect agreement. Indeed, all these expected agreements serve as checks on the correctness and accuracy of the integration procedure.

But now look at the remaining results listed in Eqs. (A5) through (A7), and compare them with their counterparts as given by Eqs. (3.12), (3.16), and (3.17). Evidently, they bear little resemblance to each other. Specifically, the natural chromaticities as given by Eq. (A6) are very different from the true natural values given by Eq. (3.16). This discrepancy is conclusive evidence that nonlinear terms in the equations of motion for dipoles are very important for chromaticities in the PSR. It is reasonable to expect that similar conclusions would hold for other rings of small or modest size. In particular, methods of chromaticity calculation which treat dipoles in the linear transfer matrix approximation are not expected to be correct for small rings.

It is interesting to note that the effect of the nonlinear dipole contributions is to raise the chromaticities. That is, the chromaticities given by Eq. (3.16) are larger than those given by Eq. (A6). Perhaps this is why the natural chromaticity of a small ring can even be positive in some regions of the tune diagram as discussed in section 3 .

At this point it is worthwhile to make a final comment which is applicable to the case of a combined function lattice. Using cylindrical coordinates and employing the polar angle as an independent variable, it can be shown that, under quite general conditions, the Hamiltonian for motion in a combined function bend can be writ-

\begin{tabular}{|c|c|c|c|c|}
\hline \multicolumn{2}{|c|}{$\begin{array}{l}\text { Horizontal Defocus Quad Strength } \\
\text { Horizontal Focus Quad Strength } \\
\text { All Sextupole Strengths }\end{array}$} & $\begin{array}{l}=-2.68 \mathrm{Tesla} / \mathrm{m} \\
=1.95 \mathrm{Tesla} / \mathrm{m} \\
=0\end{array}$ & \multirow[b]{2}{*}{$\begin{array}{c}T_{h} \\
2.2540596\end{array}$} & \multirow[b]{2}{*}{$\begin{array}{c}T_{\nu} \\
2.2499258\end{array}$} \\
\hline $\begin{array}{l}\delta \\
0\end{array}$ & $\begin{array}{l}Q_{y} \\
0\end{array}$ & $\begin{array}{c}P_{y} \\
0\end{array}$ & & \\
\hline $10^{-3}$ & $0.36732048-04$ & $-0.3355084 \varepsilon$ & 2.2520126 & 2.2480807 \\
\hline$-10^{-3}$ & -0.36694476 & 0.33514 & & 2.2517761 \\
\hline $10^{-4}$ & 0.3 & -0.33534 & 2.25 & 2.2497411 \\
\hline$-10^{-4}$ & $0.36711391-05$ & $0.33530826-04$ & 2.2542646 & 2.2501106 \\
\hline
\end{tabular}
ten in the form ${ }^{4}$

$$
\begin{array}{r}
K=\rho\left[\left(p^{0}\right)^{2}(1+\delta)^{2}-p_{\rho}^{2}-p_{z}^{2}\right]^{1 / 2} \\
-q \rho A_{\phi} .
\end{array}
$$

Here $q$ is the charge of the particle in question,

TABLE VII

Selected Closed Orbit Data for PSR with Normal Entry Bend Magnets in the Quadratic Approximation for Bends 
and the vector potential $\mathbf{A}$ is assumed to have only a $\phi$ component. Now suppose that $A_{\phi}$ is required to describe a magnetic field in a bend which contains quadrupole (and perhaps higher multipole) terms as well as a dipole term. From the relation $\mathbf{B}=\nabla \times \mathbf{A}$ and the requirement that the magnetic field $\mathbf{B}$ must be curl free, it follows that the quantity $\rho A_{\phi}$ must satisfy the equation

$$
\left(\partial_{z}^{2}+\partial_{\rho}^{2}-\rho^{-1} \partial_{\rho}\right)\left[\rho A_{\phi}(\rho, z)\right]=0 .
$$

Since the quantity $\left(\rho A_{\phi}\right)$ appears in the Hamiltonian Eq. (A8) as an effective potential, what is needed in the spirit of this appendix is an expansion of $\left(\rho A_{\phi}\right)$ within the bend magnet. For this purpose it is convenient to write

$$
\begin{aligned}
\rho & =\rho_{0}+\xi, \\
\rho A_{\phi}(\rho, z) & =f(\xi, z),
\end{aligned}
$$

where, as before, $\rho_{0}$ is the radius of the on-momentum design orbit in a bend.

Next suppose that $f$ is expanded in a double power series in $\xi$ and $z$. Without loss of generality, the various terms may be grouped together according to degree. Therefore, $f$ may be written in the form

$$
f=\sum_{n} R_{n}(\xi, z)
$$

where each $R_{n}$ is a homogeneous polynomial of degree $n$. Further, midplane symmetry is assumed so that $f$ is an even function of $z$. Then, imposition of the condition Eq. (A9) and the use of a suitable gauge shows that the first few of the $R_{n}$ are given by the expressions

$$
\begin{aligned}
R_{0}= & 0 \\
R_{1}= & B_{z}{ }^{0} \rho_{0} \xi \\
R_{2}= & B_{z}{ }^{0} \xi^{2} / 2+a_{2}\left(\xi^{2}-z^{2}\right) \\
R_{3}= & \left(a_{2} / \rho_{0}\right) \xi^{3} / 3+a_{3}\left(\xi^{3}-3 \xi z^{2}\right) \\
R_{4}= & -\left(a_{2} / \rho_{0}{ }^{2}\right)\left(\xi^{4} / 12\right)+\left(a_{3} / \rho_{0}\right)\left(\xi^{4}-z^{4}\right) / 4 \\
& +a_{4}\left(\xi^{4}-6 \xi^{2} z^{2}+z^{4}\right) .
\end{aligned}
$$

Here the coefficients $B_{z}{ }^{0}, a_{2}, a_{3}, a_{4}$, etc. are arbitrary, and their values specify the strengths of the various multipole components of the field.

Now suppose that the field in the bend magnet has a gradient so that $a_{2} \neq 0$. Then it follows from Eq. (A12) that the cubic polynomial $R_{3}$ cannot vanish no matter what the value of $a_{3}$ is. (Also, all the higher $R_{n}$ differ from zero as well. Similarly, the incorporation of a sextupole term, $a_{3} \neq 0$, into a bend field forces all the $R_{n}$ to differ from zero.) Indeed, the value of $R_{3}$ can be very large if the radius of curvature $\rho_{0}$ is sufficiently small.

Since $R_{3}$ is cubic, its effect on chromaticities is similar to that of a sextupole. The conclusion to be drawn is that methods of chromaticity calculation which treat combined function bends in the linear transfer matrix approximation are doubly cursed for small rings. That is, both the cubic terms Eq. (A3) and $R_{3}$ are ignored in such calculations, and both terms are potentially important.

\section{APPENDIX B Fringe-Field Effects}

The purpose of this appendix is to illustrate the importance of treating fringe field effects in a manner which goes beyond the usual matrix formulation. For simplicity, only the case of normal entry bend magnets is discussed. Results are similar for the case of parallel faced bends.

Consider the transition from the short straight section into a normal entry bend magnet. See Figs. 2 and 4. From a computational view point, it is easiest to treat fringe focussing as occuring in the cartesian coordinate system just before the face of the bend magnet. Let the quantities $Q_{y}{ }^{b}$, $Q_{z}{ }^{b}, P_{y}{ }^{b}, P_{z}{ }^{b}$ denote dimensionless phase-space coordinates on a trajectory just before entry into the fringe field region, and let $Q_{y}{ }^{a}, Q_{z}{ }^{a}, P_{y}{ }^{a}, P_{z}{ }^{b}$ denote their values after passage through the fringe field. The effect of entry fringe focussing is taken to be represented by the relations ${ }^{4}$

$$
\begin{aligned}
Q_{y}{ }^{a}= & Q_{y}{ }^{b}+(1 / 2)\left(Q_{z}{ }^{b}\right)^{2}(1+\delta)^{2} \\
& \times\left[(1+\delta)^{2}-\left(P_{y}{ }^{b}\right)^{2}\right]^{-3 / 2}\left(C / \rho_{0}\right) \\
P_{y}{ }^{a}= & P_{y}^{b} \\
Q_{z}{ }^{a}= & Q_{z}^{b} \\
P_{z}{ }^{a}= & P_{z}^{b}-Q_{z}{ }^{b} P_{y}{ }^{b} \\
& \times\left[(1+\delta)^{2}-\left(P_{y}{ }^{b}\right)^{2}\right]^{-1 / 2}\left(C / \rho_{0}\right) .
\end{aligned}
$$


A word is in order about the derivation and meanings of Eqs. (B1). Equations (B1b) and (B1c) specify that the horizontal momentum and vertical position of a trajectory are unchanged by passage through an entry fringe field. Thus, in this aspect, the fringe field region is treated as vanishingly short. Moreover, according to Eq. (B1d), the change in vertical momentum is proportional to the quantity

$$
P_{y}\left[(1+\delta)^{2}-\left(P_{y}^{b}\right)^{2}\right]^{-1 / 2}\left(C / \rho_{0}\right) .
$$

The factor $\left(C / \rho_{0}\right)$ arises from the introduction of dimensionless coordinates. In the usual treatment of fringe focussing, the tangent of the entrance angle as given by the ratio $v_{y} / v_{x}$ appears. ${ }^{8}$ Here $v_{x}$ is the velocity component normal to the face of the bend magnet, and $v_{y}$ is the velocity component perpendicular to the face of the bend magnet and lying in the up-down symmetry plane. A simple computation gives the result

$$
\begin{aligned}
v_{y} / v_{x} & =p_{y} / p_{x} \\
& =P_{y}\left[(1+\delta)^{2}-P_{y}^{2}-P_{z}^{2}\right]^{-1 / 2} .
\end{aligned}
$$

The quantity $\boldsymbol{P}_{z}^{2}$ is small and of second order. In particular, it is zero for all closed orbits in the case of midplane symmetry. Its neglect leads to the approximation

$$
v_{y} / v_{x} \simeq P_{y}\left[(1+\delta)^{2}-P_{y}^{2}\right]^{-1 / 2} .
$$

And by the comments just made, its neglect has no effect on the tunes of either on or off-momentum closed orbits.

With this background, it is evident that Eq. (B1d) is consistent with the usual treatment of vertical fringe field focussing. Note that for simplicity, Eq. (B1d) contains no finite gap correction, although to do so would not introduce much more complication.

What about Eq. (B1a)? In the usual treatment of a vanishing small fringe field region, it is customary to assume that the horizontal momentum component is unchanged by passage through the fringe field region. That is, the second term on the right hand side of Eq. (B1a) is omitted. However, whatever happens in the fringe field region, its effect must be such that the initial and final phase space coordinates are related by a canonical transformation. Consequently, the Poisson bracket $\left[Q_{y}{ }^{a}, P_{z}{ }^{a}\right]$ must be zero. If Eq. (B1d) is assumed to be correct, then the correction term in Eq. (B1a) is required to assure that the overall transformation is canonical. Note that the quantity $Q_{z}$ is zero on all closed orbits in the case of midplane symmetry. This observation, and the fact that $Q_{z}{ }^{b}$ appears quadratically in Eq. (B1a), means that the correction term has no effect on the tunes of either on or off-momentum closed orbits.

We are now prepared to comment on the importance of the fact that fringe field focussing depends on the angles of entry and exit, and these angles in turn depend on momentum because the shape of the design orbit depends on momentum.

The neglect of this effect amounts to replacing the quantity $v_{y} / v_{x}$ given in Eq. (B4) by its value on the on-momentum design orbit $(\delta=0)$. In the case of normal entry dipoles, the ratio $v_{y} / v_{x}$ is zero for the design orbit. In the case of parallel faced dipoles, the ratio has the value $\tan \left(18^{\circ}\right)$ for the design orbit. When this replacement is made, Eq. (B1d) is altered to become the linear relation

$$
P_{z}^{a}=P_{z}^{b}-Q_{z}^{b}\left(C / \rho_{0}\right)\left(v_{y} / v_{x}\right) \mid \begin{gathered}
\text { design } \\
\text { orbit }
\end{gathered}
$$

Also, the correction term appearing on the right of Eq. (B1a) should be deleted for consistency. The net effect of these modifications is that fringe field focussing for all trajectories is now described by the usual linear transfer matrix approximation.

Table VIII shows selected orbit data for the normal entry lattice calculated in the approximation that fringe field focussing is described by the linear transfer matrix approximation. Comparison with Table II shows that the tunes of the on-momentum design orbit are unaffected by this approximation as would be expected. Also the horizontal tunes of the off-momentum closed orbits are unaffected as would also be expected. However, the vertical off-momentum tunes differ in the fourth significant figure.

When these modified tune values are used to compute the vertical chromaticity, the result is the value $T_{v}{ }^{\prime}(0)=-1.6053$. Comparison with the correct value $T_{v}{ }^{\prime}(0)=-1.2847$ given in Eq. (3.16) shows that the use of the linear transfer matrix approximation for fringe focussing introduces significant errors.

In the case of the parallel faced bend magnet lattice, the discrepancy is also significant. For the quadrupole settings of Table $\mathrm{V}$, calculation shows that the use of the linear transfer matrix 
TABLE VIII

Selected Closed Orbit Data for PSR with Normal Entry Bend Magnets in the Linear Transfer Matrix Approximation for Fringe Field Focussing

\begin{tabular}{lcccc}
\hline Horizontal Defocus Quad Strength & $=$ & $-2.68 \mathrm{Tesla} / \mathrm{m}$ & & \\
Horizontal Focus Quad Srength & $=$ & $1.95 \mathrm{Tesla} / \mathrm{m}$ & & \\
All Sextupole Strengths & $=$ & & & \\
\multicolumn{5}{l}{} \\
$\delta$ & $Q_{y}$ & $P_{y}$ & $T_{h}$ & $T_{v}$ \\
0 & 0 & 0 & 2.2540596 & 2.2499258 \\
$10^{-3}$ & $0.36722627-04$ & $-0.33552032-03$ & 2.2529848 & 2.2483227 \\
$-10^{-3}$ & $-0.36703898-04$ & $0.33513256-03$ & 2.2551372 & 2.2515333 \\
\hline
\end{tabular}

approximation produces a vertical chromaticity value of $T_{v}{ }^{\prime}(0)=-1.5781$. Reference to Eq. (3.19) shows that the correct value should be $T_{v}{ }^{\prime}(0)=-2.11135$.

The conclusion to be drawn from these two examples is that methods of chromaticity calculation which use the linear transfer matrix approximation for fringe focussing may give significant errors. This is true even in the case of normal entry and exit where one might have thought there would be no fringe focussing effects. The subtlety of the whole matter of fringe focussing suggests that it would be valuable to study the effect of realistic fringe fields on chromaticity and to make comparisons with the hard edge model employed in this paper.

\section{REFERENCES}

1. J. Gareyte and F. Sacherer, Proceedings of the 9th International Conference on High Energy Accelerators, Stanford Linear Accelerator Center, Stanford California, 1974, p. 341; L. J. Laslett, V. K. Neil, and A. M. Sessler, Rev. Sci. Instr. 36, 436 (1965); J. D. Lawson, The Physics of Charged Particle Beams (Clarenden Press, Oxford, 1977); C. Pellegrini, Nuovo Cim. 64A, 447 (1969); F. Sacherer, Proceedings of the 9th International Conference on High Energy Accelerators, Stanford Linear Accelerator Center, Stanford California, 1974, p. 347; F. Sacherer, Methods for Computing Bunched-Beam Instabilities, CERN/SI-BR/ 72-5, CERN, Geneva, 1972; M. Sands, The Head-Tail Effect: An Instability Mechanism in Storage Rings, SLAC TN-69-8, Stanford, 1969; B. Zotter, and F. Sacherer, Transverse Instabilities of Relativistic Particle Beams in Accelerators and Storage Rings, Theoretical Aspects of the Behavior of Beams in Accelerators and Storage Rings, CERN 77-13, p. 175, CERN, Geneva, 1977.

2. A. Ando and E. Takasaki, Jpn. J. Appl. Phys. 19, 1017 (1980); W. H. Backer, Some Aspects of the Orbits in an Electron Storage Ring Used as a Synchrotron Radiation Source, Eindhoven University of Technology Thesis, 1979; W. H. Backer, C. J. Corsten, and H. L. Hagedoorn, IEEE Trans. Nucl. Sci. NS-26, 3607 (1979); R. A. Beck, R. Belbeoch, G. Gendreau, and G. Leleux, Shifts in Betatron Frequencies Due to Energy Spread, Betatron Am- plitudes and Closed Orbit Excursions, Proceedings of the Sixth International Conference on High-Energy Accelerators, Cambridge, A-63, 1967; V. A. Berezhnoi, L. I. Zaidina, N. I. Kuz'mina, R. A. Mescherov, and V. S. Rybalko, Trudy Radiotekhnicheskogo Instituta AN SSSR 30 (1977); H. Bruck, Lectures on Nonlinear Betatron Oscillations, Los Alamos National Laboratory, 1978; S. Chen, S. Fang, and C. Zhang, Chromaticity in Strong Focusing Accelerator and Storage Ring, Institute of High Energy Physics Academia Sinica report BPS/TH/81-5, 1981; C. Corsten and P. Backer, Some Aspects on Linear and Nonlinear Orbit Motion in Storage Rings, PAMPUS 79/2, Eindhoven University of Technology; M. Donald, P. Morton, and H. Wiedemann, IEEE Trans. Nucl. Sci. NS-24, 1200 (1977); P. Faugeras, A. Faugier, J. Gareyte, and A. Hilaire, Second Order Effects in the Sextupole Corrected SPS Lattice, CERN SPS/AC/79-8, 1979; A. Garren and A. Noda, A Sextupole Magnet Correction System for TARN, INS-NUMA-14, 1979; G. Guignard, First Order Chromatic Perturbations and Sextupole Strength, CERN ISR-TH/8214 and LEP Note 391, 1982; J. Jager, and D. Mohl, Comparison Methods to Evaluate the Chromaticity in LEAR, PS/DL/LEAR/Note 81-7, 1981; M. Month, Shift of the Tune Value with Sextupoles, BNL-AGSCD-37, Brookhaven National Laboratory, 1969; P. Morton, Derivation of Non-Linear Chromaticity by Higher Order Smooth Approximation, PEP-221, Stanford, 1976; A. Noda, A. Garren, T. Hori, et al., IEEE Trans. Nucl. Sci. NS-28, 2612 (1981); A. Noda, T. Hori, K. Chida, et al., Measurement of Tune Values for TARN by the RF Knock-out Method, INS-NUMA-27, 1980; H. Petit, Experiences avec des Hexapoles sur l'Anneau de Collisions d'Orsay (ACO), LAL RI/74-3, 1974; R. Servranckx, Particle Accelerators 6, 83 (1975); R. V. Servranckx and K. L. Brown, IEEE Trans. Nucl. Sci. NS-26, 3598 (1979); T. Suzuki, Tune Shifts of Betatron Oscillations and their Correction in the Synchrotron, KEK-74-6, National Laboratory for High Energy Physics, Ohomachi, Tsukuba-gun, Ibaraki-ken, Japan, 1974; T. Suzuki, Hamiltonian Formalism for the Study of Closed Orbits and Betatron Oscillations in a Perturbed Machine, KEK-78-14, National Laboratory for High Energy Physics, Oho-machi, Tsukuba-gun, Ibarakiken, Japan, 1978; H. Wiedemann, Chromaticity Correction in Large Storage Rings, PEP-220, Stanford, 1976.

3. V. S. Rybalko and N. K. Kaminsky, Trudy Radiotekhnicheskogo Instituta 19, 8 (1974).

4. Complete details concerning coordinate systems, suitable variables, equations of motion, etc. are given in A. J. Dragt, Exact Numerical Calculation of Chromaticity in Small Rings (Detailed Version), U. of Maryland Dept. of 
Physics Publication No. 81-115, 1981. Details on methods of numerical integration may be found in A. J. Dragt, Lectures on Classical Mechanics, unpublished.

5. For a discussion of variational equations, see A. J. Dragt, Lectures on Nonlinear Orbit Dynamics, Physics of High Energy Particle Accelerators (AIP Conference Proceedings, \#87) R. A. Carrigan et al., (Eds.), pps. 251-252, 1982.

6. Ibid., pgs. 249-255.
7. Ibid., pgs. 255-260.

8. C. Bovet, R. Gouiran, I. Gumowski, and K. H. Reich, A Selection of Formulae and Data Useful for the Design of A. G. Synchrotrons, CERN/MPS-SI/Int. DL/70/4, 1970; K. G. Steffen, High Energy Beam Optics (Interscience, New York 1965); H. A. Enge, Deflecting Magnets, Focusing of Charged Particles, Vol II, A. Septier, Ed. (Academic Press, New York, 1967). 